\title{
Determination of Diagnosis and Treatment for Head and Neck Cancer Patients Considering Frailty
}

\author{
Seong-Chul Yeo ${ }^{1}$ and Seung Hoon Woo ${ }^{2}$ \\ ${ }^{1}$ Department of Otorhinolaryngology, Gyeongsang National University School of Medicine, Jinju; and \\ ${ }^{2}$ Department of Otorhinolaryngology-Head and Neck Surgery, Dankook University School of Medicine, Cheonan, Korea
}

\section{노쇠 정도를 통한 두경부 암환자의 진단 및 치료 방향 결정에 관하여}

\author{
여 성 철 ${ }^{1} \cdot$ 우 승 훈 ${ }^{2}$ \\ 경상대학교 의과대학 이비인후과학교실, ${ }^{1}$ 단국대학교 의과대학 이비인후과학교실 ${ }^{2}$
}

\author{
Received January 29, 2019 \\ Revised February 21, 2019 \\ Accepted February 28, 2019 \\ Address for correspondence \\ Seung Hoon Woo, MD, PhD \\ Department of Otorhinolaryngology- \\ Head and Neck Surgery, \\ Dankook University \\ School of Medicine, \\ 201 Manghyang-ro, Dongnam-gu, \\ Cheonan 31116, Korea \\ Tel $+82-41-550-1781$ \\ Fax $+82-41-550-7837$ \\ E-mail lesaby@hanmail.net
}

As population ages, much more elderly patients have been diagnosed with head and neck cancer especially in Korea. Diverse factors such as comorbidities and disabilities make the establishment of evidence-based guidance more challenging. Chronological age, literally measured in days, months and years from the time the individual was born, is not an ideal predictor in determining personal health condition; individualization of treatment protocols with identifying the concept of frailty is the key to produce an advanced treatment plan of elderly patients suffering from head and neck malignancy. Frailty is a type of geriatric syndrome which refers to the status with age-associated declines in physiologic function and increased vulnerability to stress conditions independent of age. Therefore, screening the status of frailty may be beneficial for the elderly patients to determine decent treatment option and maximize the efficiency. In order to provide otolaryngologists prognostic information adapting the concept of frailty, research papers published from 2000 to 2018 are comprehensively reviewed in this article. For optimal care of elderly patients with head and neck cancer, frailty is definitely required to be assessed in order to establish an ideal treatment option and increase the efficacy of the treatment.

Korean J Otorhinolaryngol-Head Neck Surg 2019;62(3):141-50

Key Words Cancer · Elderly · Frailty.

\section{서 론}

우리나라의 인구 고령화는 선진국에서도 경험하지 못한 속도로 빠르게 진행되고 있어 노쇠가 임상적으로 중요한 문 제로 대두되고 있으며, 인구의 고령화로 인하여 노인 환자에 서의 두경부암 진단도 늘어나고 있다. 노인이 가지고 있는 동 반질환, 장애, 다양한 투약력, 인지력 및 사회적 이슈 등의 요 인으로 인하여 그들을 치료하는 것은 쉽지 않은 문제이다. 이들 노인 환자의 치료와 관련된 주된 치료 패러다임의 변화

This is an Open Access article distributed under the terms of the Creative Commons Attribution Non-Commercial License (https://creativecommons.org/licenses/by-nc/4.0) which permits unrestricted non-commercial use, distribution, and reproduction in any medium, provided the original work is properly cited.
중 하나는 그 동안 전반적인 건강 상태와 암 치료에 있어 불 량한 예후로 여겨지는 절대적인 나이만을 고려한 치료 프로 토콜에서 개별화된 치료 접근으로의 변화이다.

건강한 환자와 노쇠한 환자를 구분하는 것은 개개인에 따 른 예후 정보를 분석하여 치료계획을 세우는데 있어 핵심적 단계이다. 나이와 무관하게 노쇠는 복잡한 노인성 증후군으 로, 외부 스트레스에 대한 저항력이나 예비 여력이 감소하여 여러 생리적인 체계의 누적된 쇠퇴를 가져오고 작은 충격에 의해서도 합병증에 이환되기 쉬운 상태라고 할 수 있다. 이 는 기존의 다양한 연구를 통해 수술을 받는 암환자에 있어 수술후의 결과 및 치료 결정에 다양한 영향을 미치는 것으 로 알려져 있다. 더욱이 노쇠 정도를 파악함으로써 환자 개 
개인에 대한 포괄적 평가를 통해 보다 나은 결과를 얻기 위 한 조기 치료 방침의 결정도 가능하다. 특히 노인 두경부암 환자는 흡연 및 음주와 관련된 위험 요소와 밀접한 관련이 있으며, 상부 기도 및 위장관의 기능에 영향을 미치는 부위 에 종양이 발생하기 때문에 이에 따른 특징적 취약성을 보인 다. 연하과 호흡의 기능 장애는 연하 곤란, 영양 실조 및 흡인 을 야기하여 악액질, 치과적 질환, 알코올 중독 및 경제적 스 트레스로 인해 불량한 건강상태로 고통 받는 사람들에게 추 가적인 위험 요인으로 작용한다. 따라서 고령의 두경부암 환 자의 위험 요소 파악 및 치료 계획 수립에 있어 노쇠를 확인 하고 평가하는 것은 필수적이다.

\section{본 론}

\section{노쇠: 개념과 유병률}

노쇠는 점차로 고령 환자의 건강 상태를 독립적으로 결정 하는 요인으로 인식되고 있으며, 동반질환 및 장애와는 별개 또는 중복되는 요소로 고려되기도 한다. 노쇠의 정의와 관련 하여 추가적인 합의가 필요하나, 이 증후군(일련의 징후)의 개 념은 이미 확립되어있다. 노쇠는 개인의 힘, 지구력 및 신체적 능력이 감소됨으로써 적응력이 감소되고 허약함이 증가된 의학적 조건으로 특징지어진다. ${ }^{1)}$ 노쇠해지는 위험성이 있는 노인을 묘사하기 위해 노쇠징후(prefrailty)란 단어를 사용하 기도 한다. 또한 인구의 고령화로 인하여 노쇠한 상태에 놓여 있는 사람이 점차로 늘고 있다. 65 세 이상의 인구에서 노쇠가 있는 사람의 비율은 약 $10 \%$ 정도이며, 나이가 증가함에 따라 85세 이상의 인구에서는 25 50\%까지 증가한다. ${ }^{23}$ 암 그 자 체뿐 만 아니라 치료와 관련된 생리학적 요소와 연관되어 암 환자들에게서 더 높은 수준의 노쇠가 확인되었으며 약 $42 \%$ 정도로 추정 된다. ${ }^{4)}$ 지금까지 노쇠의 가설과 관련하여 두개 의 서로 다른 문헌 보고가 있다. Fried 등ㄱ은 노화와 관련된 세포 및 생리학적인 변화가 신체의 체계(systemic process) 및 항상성의 손상을 야기하여 노쇠가 나타난다고 주장하였다. Cardiovascular Health Study에 따르면 노쇠는 신체적으로 느끼는 피로감, 의도되지 않은 체중 감소, 약화된 악력, 느린 보행 속도, 저하된 신체 활동 중 3 개 이상이 존재할 때로 정 의하고 있다(Table 1). 이런 노쇠 증상의 발현은 수술과 관련
하여 가장 많이 사용되는 노쇠 측정 도구 중 하나이며 널리 검증된 방법이다.-8) 또한 Rockwood 등 ${ }^{9}$ 에 의해 개발된 결 함의 축적 이론에 따르면 의료, 사회, 기능 등의 다양한 방면 에서 여러 기능이 손실되면 노쇠가 보다 증가하게 된다고 설 명하고 있다. 누적 결함 노쇠 지수(Frailty Index of Accumulative Deficits, FI-CD)는 최대 92개의 변수를 평가한 후 모든 변수의 합계를 총 변수의 가지 수로 나누어 산출하는 지수이다. 이 지수에 따르면 증가된 결함의 누적 효과에 따 라 노쇠 정도가 증가한다는 것이 확인되었다. 평가를 위해 필요한 변수의 가짓수가 상당하기 때문에 modified Frailty Index $(\mathrm{mFI})$ 라 하는 수정본이 개발되었는데 이는 노쇠 지수 의 임상적 활용도를 향상시키기 위해 기존보다 적은 수의 변 수만으로 평가한다. ${ }^{10)}$

\section{노쇠의 진단}

노쇠의 진단을 위한 70 개 이상의 평가 방법이 문헌에 기술 되어 있으나 대부분이 충분히 검증되어있지 않으며 이들 방법 의 대부분은 앞서 기술된 노쇠 표현형(frailty phenotype) 또 는 노쇠 지수에서 유래된 것이다. 이들 방법은 의도된 목적에 따라 상이하고, 연구 환경에서 사용할 목적으로 개발된 경우 도 많으며, 선별의 목적에서부터 임상적 중재를 위한 목적의 것까지 다양하다. 또한 제한된 지역, 입원환자 혹은 수술적 치료를 받는 환자 등과 같이 특정 대상만을 위한 평가가 많 기 때문에 일반화된 사용에는 주의가 필요하다. 이 방법들은 Fried 등이이 제시 한 보행속도, 악력 등과 같이 노쇠를 대변 하는 단일 항목을 통한 평가에서부터 신체적, 기능적, 인지 적 측면을 토대로 점수를 매기는 정교하고 광범위한 방법까 지 다양하다. 임상적으로 적용 가능한 이상적인 평가 방법 여부는 평가가 필요한 임상적 상황 및 필요성, 제한된 시간 내에 시행 가능성 여부 등에 달려있다. 위험 요소의 계층화 는 단일 평가를 통해 용이하게 이루어 질 수 있다. $5 \mathrm{~m}$ 의 거 리를 걷는데 걸리는 시간을 측정한 보행속도와 Time Up and $\mathrm{Go}$ 점수는 높은 이환율과 사망률의 예측 인자로 확인 되었다. ${ }^{11-13)}$ 마찬가지로 handheld dynamometer를 통해 측 정된 악력은 암환자의 합병증 및 생존율과 관련이 있다. ${ }^{14,15}$ 보다 다양한 요소, 방법, 측정범위, 대상을 포함하는 정교한 측정 도구로서 FRAIL Index, ${ }^{16)}$ Comprehensive Geriatric

Table 1. Fried frailty criteria

\begin{tabular}{ll}
\hline Weight loss & $>4.5 \mathrm{~kg}$ lost unintentionally in prior year, or unintentional weight loss of 5\% previous year's body weight \\
Weakness & Grip strength of the dominant hand (mean of three measurements): lowest 20\% (by gender, body mass index) \\
Exhaustion & Self-reported exhaustion \\
Slowness & Walking time/4.57 minutes: slowest 20\% (by gender, height) \\
Low activity & Energy expenditure, kcals/week: lowest $20 \%$, males: $<383$ kcals/week, females: <270 kcals/week \\
\hline
\end{tabular}


Frailty and Head and Neck Cancer I Yeo SC, et al.

Table 2. Summary of the frailty assessment

\begin{tabular}{|c|c|c|c|c|c|}
\hline Instrument & $\begin{array}{c}\text { Time } \\
\text { (minutes) }\end{array}$ & $\begin{array}{l}\text { No. of } \\
\text { components }\end{array}$ & Domains & Score & Comments \\
\hline $\begin{array}{l}\text { Grip strength (in kg), } \\
\text { Kilgour, et al. }{ }^{.4)}\end{array}$ & $<2$ & 1 & $\begin{array}{l}\text { Weakness: 3-second trial with } \\
\text { handheld dynamometer }\end{array}$ & $\begin{array}{l}\text { Lowest } 20 \% \text { matched } \\
\text { for sex and BMI }\end{array}$ & $\begin{array}{l}\text { Special instruments } \\
\text { required }\end{array}$ \\
\hline $\begin{array}{l}\text { Gait speed (single } \\
\text { measure), Abellan } \\
\text { van Kan, et al. }{ }^{16)}\end{array}$ & $<5$ & 1 & $\begin{array}{l}\text { Slowness: time to walk } 4 \mathrm{~m} \text { at } \\
\text { usual pace }\end{array}$ & Time $\geq 6$ seconds & $\begin{array}{l}\text { May be difficult to } \\
\text { administer, over- } \\
\text { screens for frailty }\end{array}$ \\
\hline $\begin{array}{l}\text { TUG, Robinson, } \\
\text { et al. }{ }^{11)}\end{array}$ & $<5$ & 1 & $\begin{array}{l}\text { Gait and balance: time taken } \\
\text { to rise from chair, walk } 3 \text { m, } \\
\text { turn, and return to sitting }\end{array}$ & Time $\geq 15$ seconds & - \\
\hline $\begin{array}{l}\text { SOF, Ensrud, } \\
\text { et al. }{ }^{19)}\end{array}$ & $<5$ & 3 & $\begin{array}{l}\text { Weight loss, exhaustion, unable } \\
\text { to rise from chair five times }\end{array}$ & $\begin{array}{l}\text { Frailty } \geq 2 \text { items, prefrail } \\
1 \text {, robust } 0\end{array}$ & - \\
\hline $\begin{array}{l}\text { FRAIL scale, Abellan } \\
\text { van Kan, et al. }{ }^{(6)}\end{array}$ & $<10$ & 5 & $\begin{array}{l}\text { Self-administered: fatigue, } \\
\text { resistance, ambulation, } \\
\text { illness, weight loss }\end{array}$ & $\begin{array}{l}\text { Frail } \geq 3 \text {, prefrail } 1-2 \text {, } \\
\text { robust } 0\end{array}$ & $\begin{array}{l}\text { Requires validation in } \\
\text { oncology }\end{array}$ \\
\hline $\begin{array}{l}\text { Frailty phenotype, } \\
\text { Fried, et al. }{ }^{5}\end{array}$ & $10-15$ & 5 & $\begin{array}{l}\text { Weight loss, low physical } \\
\text { activity, exhaustion, slowness, } \\
\text { weakness }\end{array}$ & $\begin{array}{l}\text { Frailty } \geq 3 \text { items, prefrail } \\
1-2 \text {, robust } 0\end{array}$ & $\begin{array}{l}\text { No psychosocial } \\
\text { assessment, special } \\
\text { instruments required }\end{array}$ \\
\hline $\begin{array}{l}\text { G8, Bellera, } \\
\text { et al. }{ }^{28)}\end{array}$ & $5-10$ & 8 & $\begin{array}{l}\text { Age, loss of appetite } \\
\text { and weight, mobility, } \\
\text { neuropsychology, BMI, } \\
\text { polypharmacy, subjective } \\
\text { health status }\end{array}$ & Frailty=score $\geq 14$ & $\begin{array}{l}\text { Developed for geriatric } \\
\text { oncology }\end{array}$ \\
\hline $\begin{array}{l}\text { CFS, Rockwood, } \\
\text { et al. }{ }^{21)}\end{array}$ & $<5$ & 9 & $\begin{array}{l}\text { Written and visual chart with } \\
\text { pictures to assign subjective } \\
\text { frailty score }\end{array}$ & Frailty $\geq 5$ & Subjective assessment \\
\hline $\begin{array}{l}\text { EFS, Rolfson, } \\
\text { et al. }{ }^{20)}\end{array}$ & $<10$ & 9 & $\begin{array}{l}\text { Cognition, health, } \\
\text { hospitalization, social support, } \\
\text { nutrition, mood, function and } \\
\text { continence }\end{array}$ & $\begin{array}{l}\text { Frailty } \geq 8 / 14 \\
\text { vulnerable } 6-7 / 14\end{array}$ & - \\
\hline $\begin{array}{l}\text { mFl, Farhat, } \\
\text { et al. }{ }^{10)}\end{array}$ & 10 & 11 & $\begin{array}{l}\text { Comorbidities, functional } \\
\text { status, impaired sensorium }\end{array}$ & $\begin{array}{l}\text { Continuous variable, } \\
\text { Frailty }>0.27\end{array}$ & - \\
\hline $\begin{array}{l}\text { VES-13, Saliba, } \\
\text { et al. }{ }^{23)}\end{array}$ & 10 & 13 & $\begin{array}{l}\text { Self-administered } \\
\text { questionnaire on physical } \\
\text { functioning }\end{array}$ & Frailty $\geq 3$ & $\begin{array}{l}\text { May be inaccurate due } \\
\text { to overestimation of } \\
\text { own competency }\end{array}$ \\
\hline $\begin{array}{l}\text { GFI, Steverink, } \\
\text { et al. }{ }^{22)}\end{array}$ & $<15$ & 15 & $\begin{array}{l}\text { Self-assessment questionnaire } \\
\text { on mobility, fitness, } \\
\text { vision, hearing, nutrition, } \\
\text { comorbidity, cognition, } \\
\text { psychosocial }\end{array}$ & Frailty $\geq 4$ & - \\
\hline $\begin{array}{l}\text { a-CGA, Overcash, } \\
\text { et al. }{ }^{35)}\end{array}$ & 5 & 15 & $\begin{array}{l}\text { Functional status, cognition, } \\
\text { depression }\end{array}$ & $\begin{array}{l}\text { Frailty=positive score } \\
\text { on } \geq 1 \text {, domains: } \geq 1 \\
\text { for function or } \leq 6 \text { for } \\
\text { cognition or } \geq 2 \text { for } \\
\text { depression }\end{array}$ & $\begin{array}{l}\text { Developed for geriatric } \\
\text { oncology }\end{array}$ \\
\hline $\begin{array}{l}\text { FI-CGA, Jones, } \\
\text { et al. }{ }^{17)}\end{array}$ & $<15$ & 20 & $\begin{array}{l}\text { Cognition, mood and } \\
\text { motivation, communication } \\
\text { (vision, hearing, speech), } \\
\text { mobility, balance, bowel and } \\
\text { bladder function, ADLs and } \\
\text { IADLs, nutrition and social } \\
\text { resources }\end{array}$ & $\begin{array}{l}\text { Frailty: mild } 0-7 ; \\
\text { moderate } 7-13 \\
\text { severe _ } 13\end{array}$ & - \\
\hline $\begin{array}{l}\text { Fl-CD, Mitnitski, } \\
\text { et al. }{ }^{8)}\end{array}$ & 30 & 70 & $\begin{array}{l}\text { Health deficits: score of } 0 \text { (no } \\
\text { deficits) to } 1.0 \text { (all deficits) }\end{array}$ & $\begin{array}{l}\text { Continuous variable, } \\
\text { Frailty }>0.25\end{array}$ & Resource intensive \\
\hline
\end{tabular}

a-CGA: abbreviated Comprehensive Geriatric Assessment, ADLs: activities of daily living, BMl: body mass index, CFS: Clinical Frailty Scale, EFS: Edmonton Frail Scale, FI-CD: Frailty Index of Accumulative Deficits, FI-CGA: frailty index derived from Comprehensive Geriatric Assessment, G8: Geriatric 8, GFI: Groningen Frailty Index, IADLs: instrumental activities of daily living, mFI: modified Frailty Index, SOF: study of osteoporotic fractures, TUG: Timed Up and Go, VES: Vulnerable Elders Survey 
Assessment(CGA)에서 유래된 Frailty Index, ${ }^{17)} \mathrm{FI}-\mathrm{CD},{ }^{18)}$ Study of Osteoporotic Fractures Index, ${ }^{19)}$ Edmonton Frail Scale, ${ }^{20)}$ Clinical Frailty Scale, ${ }^{21)}$ Groningen Frailty Indicator $^{22)}$ 및 Vulnerable Elders Survey(VES-13) ${ }^{23)}$ 등이 개발되 어 있다(Table 2). 그러므로 이러한 다양한 측정 도구의 비교 연구를 통해 폭넓은 합의가 필요한 것은 당연하다. ${ }^{24)}$ 방대한 자료를 통한 노쇠의 총합을 기반으로 하는 방법이던, 노쇠의 신체적인 면만을 기반으로 하는 방법이던지 간에 노쇠 평가 방법은 지속적으로 논란이 되는 주제로 남아있다. 그러나 임 상적으로 어떤 형태의 평가를 사용할지에 대하여 불량한 검 사 결과가 노쇠에 대하여 일관적인 결과를 보여주기 때문에 이미 어느 정도의 합의는 이루어져 있다. CGA는 노인 환자에 있어 노쇠 평가의 표준이 되고 있다. 이는 환자의 의학적, 기 능적, 심리적, 인지적, 그리고 사회적 안녕 측면을 포함하는 표준화되고 검증된 평가 방법이다(Table 3). 또한 $\mathrm{CGA}$ 의 사 용은 생존율, 신체 기능의 향상 및 일반적 의학 상담으로는 확인하기 어려운 노쇠의 확인에 도움을 주기 때문에 Interna- tional Society of Geriatric Oncology 및 National Comprehensive Cancer Network으로부터 추천되고 있다. ${ }^{25,26)}$ 전체 $\mathrm{CGA}$ 를 도입하는 것은 시간과 노력을 필요로 하며, 의료 전 문가와 노인 보건팀 사이의 협력을 통한 다양한 접근을 요하 기 때문에 CGA가 널리 이용되는 데에 방해요소가 되고 있 다. 이 문제를 해결하기 위하여 일차적으로 정확하게 노쇠 환자를 구분하고, 이차적으로 이들로 하여금 $\mathrm{CGA}$ 검사를 받도록 하여 표준화된 치료를 가능하도록 해주는 다양한 선 별검사들이 고안되었다. 위에서 언급된 다양한 노쇠 평가 도 구들 역시 이러한 목적을 위해 다방면으로 검증되었다. ${ }^{27)}$ 이 검사들의 목적은 보다 효과적이고 객관화된 평가의 제공에 있다. 따라서 이러한 접근법은 추가적인 평가가 필요한 환자 를 선별함에 있어 충분한 민감도를 가지고 불필요한 검사를 하지 않도록 충분한 특이도를 보여야 하지만, 현재까지 이 목 표에 도달하는 단일 검사법은 없다.

현재까지 고령의 종양환자에게 사용이 가능하도록 고안된 타당성이 확인되고 특성화된 검사는 거의 없다. 문헌상으로

Table 3. CGA domains and commonly used assessment tools

\begin{tabular}{|c|c|c|}
\hline CGA domain & Tools & Description \\
\hline \multirow[t]{6}{*}{ Functional status } & ADLs & $\begin{array}{l}\text { Basic functions of everyday life: bathing, dressing, toileting, continence, } \\
\text { transferring and feeding }\end{array}$ \\
\hline & Instrumental ADLs & $\begin{array}{l}\text { Use of phone, shopping, food preparation, housekeeping, laundry, } \\
\text { transportation, responsibility for own medications and finances }\end{array}$ \\
\hline & TUG & $\begin{array}{l}\text { Time to get up from an armchair, walk } 3 \mathrm{~m} \text {, turn and return to seated } \\
\text { position }\end{array}$ \\
\hline & Karnofsky performance status & Classification based on ability to perform daily tasks \\
\hline & History of falls & \\
\hline & Handgrip strength testing & Using a dynamometer in the dominant hand \\
\hline \multirow[t]{3}{*}{ Comorbidity } & $\begin{array}{l}\text { Cumulative illness rating scale } \\
\text { for geriatrics }\end{array}$ & $\begin{array}{l}\text { Used to score comorbidities in each major area of health (e.g., } \\
\text { cardiovascular, renal) }\end{array}$ \\
\hline & ACE 27 & 27-item comorbidity index for use in patients with cancer \\
\hline & Charlson comorbidity index & Index of comorbid conditions, their severity and impact on risk of death \\
\hline Cognition & Mini mental state examination & $\begin{array}{l}\text { Assessment of orientation, memory, registration, attention, language } \\
\text { and spatial relations }\end{array}$ \\
\hline \multirow[t]{2}{*}{ Mental health } & Geriatric depression scale & Self-administered questionnaire to assess depression \\
\hline & QLQ-C30 & Self-administered questionnaire to assess quality of life \\
\hline \multirow[t]{3}{*}{ Social support } & Informal support & \\
\hline & Eligibility for care resources & \\
\hline & Financial capabilities & \\
\hline \multirow[t]{3}{*}{ Nutrition } & Mini nutritional assessment & Assesses nutritional deficiency and/or malnutrition \\
\hline & Weight loss & \\
\hline & BMI & \\
\hline Geriatric syndromes & Clinical assessment & $\begin{array}{l}\text { Clinical conditions observed in older adults such as dementia, delirium, } \\
\text { depression, osteoporosis, falls, fatigue, incontinence }\end{array}$ \\
\hline Polypharmacy & Medication review & $\begin{array}{l}\text { Identifies unnecessary and/or inappropriate medications, drug } \\
\text { interactions, and adverse drug effects }\end{array}$ \\
\hline
\end{tabular}

ACE: Adult Comorbidity Evaluation, ADLs: activities of daily living, TUG: Timed Up and Go, BMI: body mass index, CGA: Comprehensive Geriatric Assessment, QLQ-C30: 30-item Quality of Life Questionnaire-Cancer 
가장 많이 연구된 검사법은 2012년도에 Bellera 등 28 에 의해 개발된 Geriatric $8(\mathrm{G} 8)$ 이며, 이 검사는 8 가지 평가 요소로 구 성되어 있다: 한가지 요소는 나이와 관련이 있으며, 나머지 7 가지는 Table 4에 해당하는 Mini Nutritional Assessment로 부터 적용하였다(Table 4). 각 점수는 가장 허약한 상태인 0 점부터 매우 건강한 상태인 17 까지로 구성되며, 14 점을 기준

Table 4. G8 questionnaire

\begin{tabular}{|c|c|}
\hline Items & Score \\
\hline \multirow{3}{*}{$\begin{array}{l}\text { Has food intake declined } \\
\text { over the past } 3 \text { months due } \\
\text { to loss of appetite, digestive } \\
\text { problems, chewing or } \\
\text { swallowing difficulties? }\end{array}$} & $\begin{array}{l}\text { 0: Severe decrease in food } \\
\text { intake }\end{array}$ \\
\hline & $\begin{array}{l}\text { 1: Moderate decrease in } \\
\text { food intake }\end{array}$ \\
\hline & 2: No decrease in food intake \\
\hline \multirow{4}{*}{$\begin{array}{l}\text { Weight loss during the last } \\
3 \text { months }\end{array}$} & $0:$ Weight loss $>3 \mathrm{~kg}$ \\
\hline & 1: Does not know \\
\hline & $\begin{array}{l}\text { 2: Weight loss between } 1 \text { and } \\
3 \mathrm{~kg}\end{array}$ \\
\hline & 3: No Weight loss \\
\hline \multirow[t]{3}{*}{ Mobility } & 0: bed or chair bound \\
\hline & $\begin{array}{l}\text { 1: Able to get out of bed/ } \\
\text { chair but does not go out }\end{array}$ \\
\hline & 2: Goes out \\
\hline \multirow[t]{3}{*}{$\begin{array}{l}\text { Neuropsychological } \\
\text { problems }\end{array}$} & $\begin{array}{l}\text { 0: Severe dementia or } \\
\text { depression }\end{array}$ \\
\hline & $\begin{array}{l}\text { 1: Mild dementia or } \\
\text { depression }\end{array}$ \\
\hline & 2: No psychological problems \\
\hline \multirow{4}{*}{$\begin{array}{l}\text { BMI (weight in } \mathrm{kg} / \text { height } \\
\text { in } \mathrm{m}^{2} \text { ) }\end{array}$} & $0: \mathrm{BMI}<19$ \\
\hline & 1: BMI: 19-BMI <21 \\
\hline & 2: $\mathrm{BMI}: 21-\mathrm{BMI}<3$ \\
\hline & 3: $\mathrm{BMI}: 23-\mathrm{BMI}>23$ \\
\hline \multirow{2}{*}{$\begin{array}{l}\text { Takes more than three } \\
\text { medications per day }\end{array}$} & $0:$ Yes \\
\hline & $1:$ No \\
\hline \multirow{4}{*}{$\begin{array}{l}\text { In comparison with other } \\
\text { people of the same age, } \\
\text { how does the patient } \\
\text { consider his/her health } \\
\text { status? }\end{array}$} & 0: Not as good \\
\hline & 0.5: Does not know \\
\hline & 1: As good \\
\hline & 2: Better \\
\hline \multirow[t]{3}{*}{ Age (years) } & $0:>85$ \\
\hline & $1: 80-85$ \\
\hline & $2:<80$ \\
\hline Total score & $0-17$ \\
\hline
\end{tabular}

BMl: body mess index, G8: Geriatric 8
으로 추가적 $\mathrm{CGA}$ 검사 진행 여부가 결정된다. 검사 시간은 평균 5 분 정도이며, 간호사를 비롯한 여타의 검사자에 의해 시행이 되며 다양한 연구를 통하여 검증이 된 검사법이다. Kenis 등 29)은 70세 이상에서 새롭게 암으로 진단된 937명의 환자를 대상으로 G8의 타당성 및 예후 결정의 유용성을 확 인하였다. G8 검사는 $\mathrm{CGA}$ 를 통해 결정된 노쇠 환자와 비교 하여 $86.5 \%$ 의 양성 예측도를 보였으며 $61.3 \%$ 의 음성 예측도 를 보였다. 더욱이 G8은 환자의 기능 상실 및 전체 생존율 면 에 있어 의미 있는 예후 결과를 보여주었다. 또한 CGA, G8 및 VES-13을 가지고 다양한 암을 가진 1325명의 환자를 평가 하는 대규모의 다기관 연구가 진행되었고 그 결과 $\mathrm{G} 8$ 의 민감 도와 특이도는 각각 $76.5 \%$ 와 $64.4 \%$ 로 확인되었으며, 1 년 생 존율에 대한 개별 예후인자로 적합한 검사로 보고되었다. ${ }^{30)}$ 그 밖의 다양한 연구에서 $\mathrm{G} 8$ 의 민감도는 $\mathrm{CGA}$ 로 평가한 결 과와 비교하여 65 92\%정도의 민감도를 보이는 것으로 보고 하였고, 대다수에서 $80 \%$ 이상으로 확인되었으며, 특이도는 40 75\%로 보고되었다. ${ }^{31-34)}$ 또한 간소화된 abbreviated CGA (aCGA)는 정형화된 평가가 필요한 노인성 종양학 분야의 선 별 검사 수단으로 고안되었으며 G8과는 대조적으로 $\mathrm{aCGA}$ 는 $97 \%$ 의 높은 특이도를 보였으며 민감도는 상대적으로 낮 은 $51 \%$ 를 보여 이에 상응하는 목적으로 주로 사용된다. ${ }^{35,36)}$

\section{노쇠와 암}

이질적인 고령의 암 환자군에서 최적의 치료 계획을 세우 는 것은 다소 어려운 문제일 수 있다. 그들이 가지고 있는 여 러 방면의 취약성으로 인해 악성 종양의 치료 계획을 수립하 는데 있어 이완된 장기 및 연령에 기반을 두는 전통적인 접 근 방식은 제한 점이 있다. 응급수술부터 계획된 수술에 이 르기까지 환자의 노쇠는 술 후 합병증, 사망률, 입원기간 및 퇴원 후 계획을 포함한 전반적인 수술의 불량한 결과에 영향 을 미친다. ${ }^{37}$ 종약학적으로 불량한 수술 결과는 다양한 암으 로 수술을 받는 환자의 노쇠 상태와 연관이 있는 것으로 나 타났다. ${ }^{7,838-57)}$ 또한 노쇠는 항암치료 및 방사선치료에 대한 환자의 내성과도 관련이 있으며, 나아가 치료 후 부작용 발생 및 삶의 질 저하를 유발하는 것으로 알려져 있다. 결과적으 로 치료에 대한 순응 불량 및 중단은 건강 관리(비용)의 증가 와 암 치료의 위축으로 이어진다. ${ }^{58,59)}$ 이러한 목적과 관련하 여 조사한 연구에 따르면 노쇠의 평가는 항암치료의 부작용 과 치료 중단의 가능성 측면에 대해 일반적으로 사용되는 신 체 활동 점수와 비교하여 보다 우수한 예측 결과를 보여주 는 것으로 나타났다. ${ }^{60,61)}$ 유방암 환자군을 대상으로 시행한 연 구에서 Fried 노쇠 점수(Fried frailty score)와 암 특이적 CGA 검사를 통해 방사선 치료로 발생하는 신체 위약성을 예 
측할 수 있음이 확인되었다. ${ }^{62}$ 이러한 노쇠 평가는 건강한 노 인 환자를 확인하여 젊은 환자와 비슷한 항암치료 결과를 이끌어내는데 중요한 역할을 하며, 노쇠를 가진 노인 환자에 서 노인학 전문가가 개입하도록 하여 보다 나은 치료 결과를 기대할 수도 있다. ${ }^{63)}$ 노쇠의 진단은 그 자체가 치료방향에 미 치는 영향뿐만 아니라, 다학제적 치료팀으로 하여금 어떠한 추가적 노력이 필요한지에 대한 정보를 제공해주는 중요한 역 할을 한다. 다양한 종류의 암을 가진 1967명의 노인 환자군 를 포함하는 대규모 전향적 다기관 연구를 통해 CGA가 치 료 결정에 미치는 영향이 조사되었다. 해당 연구에서 치료를 담당하는 의사의 설문 조사 결과에 따르면 노쇠 평가 결과가 $25.3 \%$ 의 사례에서 치료 결정에 영향을 미친 것으로 나타났 다. ${ }^{64)}$ 그 밖의 여러 소규모 연구에서도 비슷한 결과를 보였으 며, 고령 암환자의 20 49\%의 경우에서 이를 통해 치료 방침 이 변경되었다고 보고하고 있다. ${ }^{65,66)}$ 치료 강도에 미치는 영 향을 보고한 연구에서는 $90 \%$ 이상의 경우에서 치료 강도를 낮추게 되었다는 결과가 보고되었다. 더욱이 평가가 시행된 환자의 26 83\%에 해당하는 대부분의 경우에서 노쇠로 인 한 개입이 발생하였으며, 이들을 위한 지원 방법으로 사회적 지지, 약물적 및 영양 측면의 지원이 시행되었다.

\section{노쇠와 두경부암}

비록 과거에는 환자의 연령 및 동반질환 여부가 환자의 위 험성을 판단하기 위한 주된 요소로 사용되었으나, 최근에는 노인 두경부암 환자의 노쇠 평가가 널리 보급되고 임상적 실 체로 인식이 바뀌고 있다. Pottel 등 ${ }^{34}$ 은 두경부암 환자 중 $\mathrm{CGA}$ 를 기준으로 단독 방사선치료를 시행 받은 환자의 $68.6 \%$, 항암방사선 동시 치료를 받은 환자의 $72 \%$ 에서 노쇠가 확인 되었다고 보고하였다. ${ }^{67)}$ 이들 중 가장 흔하게 노쇠가 확인된 영역은 동반질환(77 84.3\%)과 영양결핍(49 49\%)이었다. 이 러한 결과는 수술적 치료 혹은 비수술적 치료를 받은 두경부 암 환자 모두를 대상으로 포함한 연구 결과와는 다소 차이 를 보였다. 대한이비인후과학회의 Kwon 등 ${ }^{68)}$ 에 의한 연구 결과 $43.6 \%$ 의 환자에서 노쇠가 있다고 보고하였고, 두경부 의 피부암과 타액선암이 포함된 환자군을 통한 Bras 등 ${ }^{69}$ 의 보고에 따르면 약 $40 \%$ 에서 노쇠의 동반을 보고하였다. 이와 같은 상이한 결과는 연구 대상, 노쇠의 평가 방법, 선택 편향 (selection bias) 등의 요인에 의해 설명될 수 있으며, 수술적 치료를 받은 환자군 보다는 방사선 치료를 받은 환자군에서 더 과장되어 나타날 수 있다. 노쇠와 관련된 일반적인 문헌들 이 고령의 두경부암 환자에서 적용될 수도 있겠지만, 연하곤 란이나 호흡곤란과 같이 종양이 기관 및 식도에 위치하여 발 생하는 특징적 사항들로 인하여 보다 명확한 근거를 통한 고
려가 필요하다. 두경부암 환자는 종양의 물리적 영향과 이에 따른 항암 또는 방사선 치료로 인한 영향에 의해 연하장애 및 호흡곤란에 특히 취약하다. 구강, 구인두, 하인두, 그리고 후 두의 종양은 통증, 폐색 혹은 연하기전의 부조화로 인한 섭 식 감소와 기도 흡인을 조장할 수 있다. ${ }^{70)}$ 또한 항암 및 방사 선치료 도중에 발생되는 점막염, 구강건조증, 미각장애, 오연 및 식욕부진으로 인해 더 큰 영양 문제가 야기될 수 있다. 이 러한 문제에 보다 빠르게 접근하는 것은 음식물 섭취 및 영 양상태를 개선시키고 치료 전 노쇠를 최적화 시키는 조치를 가능하게 하여 적절한 시기에 치료를 시작하는데 도움이 될 수 있다. ${ }^{71)}$

\section{두경부암 환자에서의 노쇠의 선별}

두경부암 환자군에서 흔히 사용되는 검사 도구의 타당성 을 입증하기 위한 두 연구가 기존에 시행되었다. 한 대규모의 전향적 다기관 연구에서는 노쇠 환자를 선별하기 위한 도구 로서의 ONCODAGE의 타당성을 평가하였고, ${ }^{30)}$ 또 다른 연 구에서는 CGA 검사 시행이 필요한 환자의 선별 및 1 년 생존 율의 예후 확인 수단으로서의 G8의 검증이 시행되었다. 두경 부 암환자의 소그룹 분석에서 G8은 노쇠를 판단하는데 있 어 $94.1 \%$ 의 민감도와 $83.3 \%$ 의 특이도(양성 예측도: $98.5 \%$, 음성 예측도: $55.6 \%$ )의 결과를 나타냈다. 또한 동반된 항암 치료 여부와 상관 없이 치료적 목적의 1차 또는 추가 방사선 치료를 받은 환자군에서 G8과 VES-13을 평가하기 위한 예 비연구를 통해 두 가지 검사 모두 양호한 진단적 가치가 확인 되었다. ${ }^{34)} \mathrm{VES}-13$ 은 민감도와 특이도가 각각 $57.1 \%$ 와 $100 \%$ 로 확인되었고, G8의 경우 $85.7 \%$ 의 민감도와 $75 \%$ 의 특이도 를 보였다. 더욱이 두 검사의 조합을 통해 보다 향상된 진단 적 효용성이 확인되었다. G8은 두경부암 환자에서 흔히 발생 하는 영양과 관련된 부분에 더 많은 가중치를 두었기 때문 에 보다 우수성이 있을 것으로 사료된다. 이는 질량 효과가 큰 진행된 병기에서 보다 높은 연하장애가 발생하며, 이 환자들 에서 보다 높은 노쇠가 확인되는 경향성에 의해 뒷받침된다. 이 때문에 상부 기관식도의 질환으로 인한 고유한 예후 인자 를 확인하여 고령의 두경부암 환자군에 적용 가능한 새로운 선별평가 도구를 고안하려는 노력이 진행되고 있다. Kwon 등 ${ }^{68}$ 은 호흡 및 후두 기능의 지표로 삼기 위해 CGA 평가 항목 에 1초당 노력성 호기 용적 및 voice handicap index(VHI) 를 함께 추가하였고, 삼킴 기능을 평가하기 위해 MD Anderson Dysphagia Inventory(MDADI) 및 videofluoroscopic swallow studies 점수를 추가하였다. 또한 저작이나 삼킴에 미치는 문제를 파악하고자 치과적 접근을 하였다. $\mathrm{CGA}$ 는 2 년 이환율과는 관계가 없는 것으로 밝혀졌기에 삼킴과 호흡 
기능을 측정하는 지표를 활용한 9 개의 측면을 포함하는 두 경부암 특이적 선별 도구를 개발했으며, 이는 이환율과 사망 률의 예측에 영향을 미친다는 것이 밝혀졌다. 불량한 결과와 관련된 것으로 알려진 독립적인 요인들이 있는데 이는 75세 이상의 고령, 심각한 흡연, 체중감소, Eastern Cooperative Oncology Group status $\geq 2$, Charlson Comorbidity Index $\geq 1$, 치과적 문제, $\mathrm{VHI} \geq 8$, MDADI $<70$, 그리고 Beck Depression Inventory $\geq 14$ 이다. 이를 토대로 점수가 5 9에 해 당하는 고위험군은 2 년 이환율 및 사망률 면에서 보다 높은 위험성을 보였다.

\section{두경부암 환자의 노쇠와 치료 결과}

두경부암 환자에 있어 노쇠는 불량한 치료 결과, 건강과 관련된 삶의 질 및 치료 부작용 등과 밀접한 관련이 있는 것 으로 알려져 있다. 다양한 두경부암으로 수술을 받은 환자를 대상으로 환자의 노쇠 여부 및 이와 관련된 진단 및 치료 결 과의 상관성 확인을 위한 연구가 시행되었다. 그 결과 환자의 7.4\%에서만 노쇠가 있는 것으로 확인되었으나, 환자의 노쇠 는 수술 후 부작용, 약물 합병증, 원내 사망, 입원 기간 그리 고 입원 비용과 밀접한 상관관계가 있는 것으로 드러났다. ${ }^{72)}$ Abt 등기는 두경부 수술을 받은 환자 1192 명의 술 후 결과를 $\mathrm{mFI}$ 를 이용하여 후향적으로 분석하였다. 위약성이 있다고 판단되는 환자에서 전반적인 이환율과 사망률의 증가를 보 였으며, 특히 의도하지 않았던 추가적 수술 등을 포함한 술 후 합병증에 유의미한 영향을 미침이 확인되었다. 또한 하위 그룹 분석에서 하악절제술, 설절제술, 후두절제술을 시행 받 은 환자의 경우 $\mathrm{mFI}$ 점수가 증가함에 따라 Clavien-Dindo grade IV에 해당하는 심각한 합병증의 발생의 유의미하게 증 가하였다. Kwon 등 ${ }^{68)}$ 은 수술적 또는 비수술적 치료를 받은 두경부암 환자를 대상으로 $\mathrm{CGA}$ 를 통해 진단된 노쇠와 2년 사망률과의 관계를 분석하였으나 특별한 상관관계는 확인되 지 않았다. 그 밖에 Pottel 등 ${ }^{34)}$ 은 수술을 받지 않은 두경부 환자군에서 G8 결과가 36 개월 생존율의 독립적 예측 인자임 을 보고하였다. ${ }^{67)}$ 또한 이들은 건강과 관련된 삶의 질이나 치 료 독성 측면에 대한 추적 연구를 추가적으로 시행하였다. ${ }^{74)}$ 그 결과에 노쇠가 있는 환자는 항암화학요법 동반여부와 무 관하게 방사선치료 전/도중/후 모두에서 삶의 질 점수가 지속 적으로 낮게 유지되는 것이 확인되었다. 또한 치료 전 $\mathrm{CGA}$ 에 서 비정상으로 확인된 영역의 수와 삶의 질 사이의 상관관계 역시 확인되었다. 고령의 환자에서도 치료가 끝남과 동시에 삶의 질 점수가 치료 전과 유사한 상태로 돌아가나, 노쇠가 동반된 환자의 경우 치료 전과 비슷하게 돌아가기 까지는 5 개 월 정도가 소요됨이 확인되었다. 추가적인 분석에서 노쇠한
환자는 그렇지 않은 사람과 비교하여 유의하게 기능 점수는 낮았고 증상 점수는 높았다. 이 데이터들은 수명보다는 삶의 질에 더 가치를 두는 노쇠가 있는 환자에서 치료 방법을 설 명할 때 치료 부작용 및 기대 여명과 관련된 균형성이 반드 시 설명되어야 함을 강조한다. 또한 방사선 치료를 받는 두경 부암 환자의 연속적 $\mathrm{CGA}$ 와 치료 독성과의 상관성과 관련된 연구가 같은 저자들에 의해 시행되었다. ${ }^{74)}$ 비록 노쇠한 환자 의 경우 더 심한 독성 및 입원 경향이 있음이 밝혀졌으나, 모 든 환자에서 $\mathrm{G} 8$ 을 기준으로 주로 영양과 관련된 측면에서의 취약성이 치료 도중 발생함이 확인되었는데, 이 결과 역시 구 강의 기능이 노쇠에 중요한 요소임이 강조되는 부분이다.

\section{결 론}

인구의 고령화와 함께 노년층에서 확인되는 노쇠는 증가하 고 있다. 여러 연구의 결과에서 일반인 뿐만 아니라 암 환자 군에서 노쇠는 여러 종류의 불량한 결과 및 예후와 관련됨 이 보고되고 있다. 두경부암 환자에서 노쇠가 갖는 중요성을 완전하게 이해하기 위해서는 보다 많은 연구가 필요하다. 비 록 CGA가 노쇠 진단을 위한 표준 검사로 알려져 있으나, G8 역시 임상에서 효과적으로 시행 가능하고 다양한 이질적 환 자군의 개별화된 치료 예후 판단에 도움을 주는 실용적이고 객관적인 검사 도구이다. 따라서 두경부 영역의 진단 및 치료 를 결정할 때 객관적인 나이만을 기준으로 하지 말고 G8 등 노쇠를 평가 할 수 있는 도구들의 사용을 강력히 권장하며, 이를 통해 두경부 질환 환자들의 문제 해결에 필요한 판단 증거 및 최적화된 치료의 개발이라는 궁극적 목표에 접근할 수 있는 새로운 지평을 열어줄 것으로 사료된다.

\section{Acknowledgments}

No financial or material support has been received for this work; moreover, the authors declare no financial interests in companies or other entities that may be interested in the results of this study.

\section{REFERENCES}

1) Morley JE, Vellas B, van Kan GA, Anker SD, Bauer JM, Bernabei $\mathrm{R}$, et al. Frailty consensus: A call to action. J Am Med Dir Assoc 2013;14(6):392-7.

2) Song X, Mitnitski A, Rockwood K. Prevalence and 10-year outcomes of frailty in older adults in relation to deficit accumulation. J Am Geriatr Soc 2010;58(4):681-7.

3) Collard RM, Boter H, Schoevers RA, Oude Voshaar RC. Prevalence of frailty in community-dwelling older persons: A systematic review. J Am Geriatr Soc 2012;60(8):1487-92.

4) Handforth C, Clegg A, Young C, Simpkins S, Seymour MT, Selby PJ, et al. The prevalence and outcomes of frailty in older cancer patients: A systematic review. Ann Oncol 2015;26(6):1091-101.

5) Fried LP, Tangen CM, Walston J, Newman AB, Hirsch C, Gottdiener $\mathrm{J}$, et al. Frailty in older adults: evidence for a phenotype. J Gerontol 
A Biol Sci Med Sci 2001;56(3):M146-56.

6) Makary MA, Segev DL, Pronovost PJ, Syin D, Bandeen-Roche K, Patel P, et al. Frailty as a predictor of surgical outcomes in older patients. J Am Coll Surg 2010;210(6):901-8.

7) Tan KY, Kawamura YJ, Tokomitsu A, Tang T. Assessment for frailty is useful for predicting morbidity in elderly patients undergoing colorectal cancer resection whose comorbidities are already optimized. Am J Surg 2012;204(2):139-43.

8) Courtney-Brooks M, Tellawi AR, Scalici J, Duska LR, Jazaeri AA, Modesitt SC, et al. Frailty: An outcome predictor for elderly gynecologic oncology patients. Gynecol Oncol 2012;126(1):20-4.

9) Rockwood K, Mitnitski A, Song X, Steen B, Skoog I. Long-term risks of death and institutionalization of elderly people in relation to deficit accumulation at age 70. J Am Geriatr Soc 2006;54(6):975-9.

10) Farhat JS, Velanovich V, Falvo AJ, Horst HM, Swartz A, Patton JH $\mathrm{Jr}$, et al. Are the frail destined to fail? Frailty index as predictor of surgical morbidity and mortality in the elderly. J Trauma Acute Care Surg 2012;72(6):1526-30; discussion 1530-1.

11) Robinson TN, Sauaia A. Reply to letter: "Slower walking speed forecasts increased postoperative morbidity and 1-year mortality across surgical specialties." Ann Surg 2015;262(2):e75-6.

12) Afilalo J, Kim S, O’Brien S, Brennan JM, Edwards FH, Mack MJ, et al. Gait speed and operative mortality in older adults following cardiac surgery. JAMA Cardiol 2016;1(3):314-21.

13) Abellan van Kan G, Rolland Y, Andrieu S, Bauer J, Beauchet O, Bonnefoy M, et al. Gait speed at usual pace as a predictor of adverse outcomes in community-dwelling older people an International Academy on Nutrition and Aging (IANA) task force. J Nutr Health Aging 2009;13(10):881-9.

14) Kilgour RD, Vigano A, Trutschnigg B, Lucar E, Borod M, Morais JA. Handgrip strength predicts survival and is associated with markers of clinical and functional outcomes in advanced cancer patients. Support Care Cancer 2013;21(12):3261-70.

15) Chen $\mathrm{CH}$, Ho-Chang, Huang YZ, Hung TT. Hand-grip strength is a simple and effective outcome predictor in esophageal cancer following esophagectomy with reconstruction: A prospective study. J Cardiothorac Surg 2011;6:98.

16) Abellan van Kan G, Rolland YM, Morley JE, Vellas B. Frailty: Toward a clinical definition. J Am Med Dir Assoc 2008;9(2):71-2.

17) Jones DM, Song $X$, Rockwood K. Operationalizing a frailty index from a standardized comprehensive geriatric assessment. J Am Geriatr Soc 2004;52(11):1929-33.

18) Mitnitski AB, Mogilner AJ, Rockwood K. Accumulation of deficits as a proxy measure of aging. ScientificWorldJournal 2001;1:323-36.

19) Ensrud KE, Ewing SK, Taylor BC, Fink HA, Stone KL, Cauley JA, et al. Frailty and risk of falls, fracture, and mortality in older women: the study of osteoporotic fractures. J Gerontol A Biol Sci Med Sci 2007;62(7):744-51.

20) Rolfson DB, Majumdar SR, Tsuyuki RT, Tahir A, Rockwood K. Validity and reliability of the Edmonton frail scale. Age Ageing 2006; 35(5):526-9.

21) Rockwood K, Song X, MacKnight C, Bergman H, Hogan DB, McDowell I, et al. A global clinical measure of fitness and frailty in elderly people. CMAJ 2005;173(5):489-95.

22) Steverink N, Slaets J, Schuurmans H, Van Lis M. Measuring frailty: Development and testing of the groningen frailty indicator (GFI). Gerontologist 2001;41:236-7.

23) Saliba D, Elliott M, Rubenstein LZ, Solomon DH, Young RT, Kamberg CJ, et al. The vulnerable elders survey: A tool for identifying vulnerable older people in the community. J Am Geriatr Soc 2001; 49(12):1691-9.

24) Theou O, Brothers TD, Mitnitski A, Rockwood K. Operationalization of frailty using eight commonly used scales and comparison of their ability to predict all-cause mortality. J Am Geriatr Soc 2013;61
(9):1537-51.

25) Ellis G, Whitehead MA, Robinson D, O'Neill D, Langhorne P. Comprehensive geriatric assessment for older adults admitted to hospital: Meta-analysis of randomised controlled trials. BMJ 2011; 343:d6553.

26) Decoster L, Van Puyvelde K, Mohile S, Wedding U, Basso U, Colloca $\mathrm{G}$, et al. Screening tools for multidimensional health problems warranting a geriatric assessment in older cancer patients: an update on SIOG recommendationsdagger. Ann Oncol 2015;26(2):288-300.

27) Hamaker ME, Jonker JM, de Rooij SE, Vos AG, Smorenburg CH, van Munster BC. Frailty screening methods for predicting outcome of a comprehensive geriatric assessment in elderly patients with cancer: A systematic review. Lancet Oncol 2012;13(10):e437-44.

28) Bellera CA, Rainfray M, Mathoulin-Pélissier S, Mertens C, Delva F, Fonck M, et al. Screening older cancer patients: first evaluation of the G-8 geriatric screening tool. Ann Oncol 2012;23(8):2166-72.

29) Kenis C, Decoster L, Van Puyvelde K, De Grève J, Conings G, Milisen K, et al. Performance of two geriatric screening tools in older patients with cancer. J Clin Oncol 2014;32(1):19-26.

30) Soubeyran P, Bellera C, Goyard J, Heitz D, Curé H, Rousselot H, et al. Screening for vulnerability in older cancer patients: the ONCODAGE prospective multicenter cohort study. PLoS One 2014;9(12):e115060.

31) Liuu E, Canouï-Poitrine F, Tournigand C, Laurent M, Chaubet M, Caillet $P$, et al. External validation of the G-8 geriatric screening tool to identify vulnerable elderly cancer patients: the ELCAPA-02 study. J Geriatr Oncol 2012;3:S45-S6.

32) Luce S, De Breucker S, Van Gossum A, Demols A, Mekinda Z, Ena $\mathrm{G}$, et al. How to identify older patients with cancer who should benefit from comprehensive geriatric assessment? J Geriatr Oncol 2012;3(4): 351-8.

33) Baitar A, Van Fraeyenhove F, Vandebroek A, De Droogh E, Galdermans $\mathrm{D}$, Mebis $\mathrm{J}$, et al. Evaluation of the groningen frailty indicator and the G8 questionnaire as screening tools for frailty in older patients with cancer. J Geriatr Oncol 2013;4:32-8.

34) Pottel L, Boterberg T, Pottel H, Goethals L, Van Den Noortgate N, Duprez F, et al. Determination of an adequate screening tool for identification of vulnerable elderly head and neck cancer patients treated with radio (chemo) therapy. J Geriatr Oncol 2012;3(1):24-32.

35) Overcash JA, Beckstead J, Moody L, Extermann M, Cobb S. The abbreviated comprehensive geriatric assessment (aCGA) for use in the older cancer patient as a prescreen: Scoring and interpretation. Crit Rev Oncol Hematol 2006;59(3):205-10.

36) Kellen E, Bulens P, Deckx L, Schouten H, Van Dijk M, Verdonck I, et al. Identifying an accurate pre-screening tool in geriatric oncology. Crit Rev Oncol Hematol 2010;75(3):243-8.

37) Lin HS, Watts JN, Peel NM, Hubbard RE. Frailty and post-operative outcomes in older surgical patients: A systematic review. BMC Geriatr 2016;16(1):157.

38) Lascano D, Pak JS, Kates M, Finkelstein JB, Silva M, Hagen E, et al. Validation of a frailty index in patients undergoing curative surgery for urologic malignancy and comparison with other risk stratification tools. Urol Oncol 2015;33(10):426.e1-12.

39) Neuman HB, Weiss JM, Leverson G, O'Connor ES, Greenblatt DY, Loconte NK, et al. Predictors of short-term postoperative survival after elective colectomy in colon cancer patients $\geq 80$ years of age. Ann Surg Oncol 2013;20(5):1427-35.

40) Hoffen J, Fahey N, Wang C, Park S. PD10-06 patient frailty predicts for serious complications after renal cancer surgery-analysis from NSQIP. J Urol 2016;195(4s):e241.

41) Mogal H, Vermilion SA, Dodson R, Hsu FC, Howerton R, Shen P, et al. Modified frailty index predicts morbidity and mortality after pancreaticoduodenectomy. Ann Surg Oncol 2017;24(6):1714-21.

42) Uppal S, Igwe E, Rice LW, Spencer RJ, Rose SL. Frailty index predicts severe complications in gynecologic oncology patients. 
Gynecol Oncol 2015;137(1):98-101.

43) Chappidi MR, Kates M, Patel HD, Tosoian JJ, Kaye DR, Sopko NA, et al. Frailty as a marker of adverse outcomes in patients with bladder cancer undergoing radical cystectomy. Urol Oncol 2016;34(6):256. e1-6.

44) Mandelblatt JS, Cai L, Luta G, Kimmick G, Clapp J, Isaacs C, et al. Frailty and long-term mortality of older breast cancer patients: CALGB 369901 (Alliance). Breast Cancer Res Treat 2017;164(1):107-17.

45) Clough-Gorr KM, Thwin SS, Stuck AE, Silliman RA. Examining five- and ten-year survival in older women with breast cancer using cancer-specific geriatric assessment. Eur J Cancer 2012;48(6):805-12.

46) Clough-Gorr KM, Stuck AE, Thwin SS, Silliman RA. Older breast cancer survivors: geriatric assessment domains are associated with poor tolerance of treatment adverse effects and predict mortality over 7 years of follow-up. J Clin Oncol 2010;28(3):380-6.

47) Kumar A, Langstraat CL, DeJong SR, McGree ME, Bakkum-Gamez JN, Weaver AL, et al. Functional not chronologic age: Frailty index predicts outcomes in advanced ovarian cancer. Gynecol Oncol 2017; 147(1):104-9.

48) Brown JC, Harhay MO, Harhay MN. The prognostic importance of frailty in cancer survivors. J Am Geriatr Soc 2015;63(12):2538-43.

49) Choi JY, Yoon SJ, Kim SW, Jung HW, Kim KI, Kang E, et al. Prediction of postoperative complications using multidimensional frailty score in older female cancer patients with American Society of Anesthesiologists physical status class 1 or 2. J Am Coll Surg 2015; 221(3):652-60.e2.

50) Cloney M, D’Amico R, Lebovic J, Nazarian M, Zacharia BE, Sisti $\mathrm{MB}$, et al. Frailty in geriatric glioblastoma patients: A predictor of operative morbidity and outcome. World Neurosurg 2016;89:362-7.

51) Choe YR, Joh JY, Kim YP. Association between frailty and readmission within one year after gastrectomy in older patients with gastric cancer. J Geriatr Oncol 2017;8(3):185-9.

52) Tegels JJ, de Maat MF, Hulsewé KW, Hoofwijk AG, Stoot JH. Value of geriatric frailty and nutritional status assessment in predicting postoperative mortality in gastric cancer surgery. J Gastrointest Surg 2014;18(3):439-45; discussion 445-6.

53) Kristjansson SR, Nesbakken A, Jordhøy MS, Skovlund E, Audisio $\mathrm{RA}$, Johannessen HO, et al. Comprehensive geriatric assessment can predict complications in elderly patients after elective surgery for colorectal cancer: A prospective observational cohort study. Crit Rev Oncol Hematol 2010;76(3):208-17.

54) Ommundsen N, Wyller TB, Nesbakken A, Jordhøy MS, Bakka A, Skovlund E, et al. Frailty is an independent predictor of survival in older patients with colorectal cancer. Oncologist 2014;19(12):1268-75.

55) Kristjansson SR, Rønning B, Hurria A, Skovlund E, Jordhøy MS, Nesbakken A, et al. A comparison of two pre-operative frailty measures in older surgical cancer patients. J Geriatr Oncol 2012;3(1):1-7.

56) Lu J, Cao LL, Zheng CH, Li P, Xie JW, Wang JB, et al. The preoperative frailty versus inflammation-based prognostic score: Which is better as an objective predictor for gastric cancer patients 80 years and older? Ann Surg Oncol 2017;24(3):754-62.

57) Robinson TN, Wallace JI, Wu DS, Wiktor A, Pointer LF, Pfister SM, et al. Accumulated frailty characteristics predict postoperative discharge institutionalization in the geriatric patient. J Am Coll Surg 2011;213(1):37-42; discussion 42-4.

58) Calhoun EA, Chang CH, Welshman EE, Fishman DA, Lurain JR, Bennett CL. Evaluating the total costs of chemotherapy-induced toxicity: Results from a pilot study with ovarian cancer patients. Oncologist 2001;6(5):441-5.

59) Butler L, Bacon M, Carey M, Zee B, Tu D, Bezjak A. Determining the relationship between toxicity and quality of life in an ovarian cancer chemotherapy clinical trial. J Clin Oncol 2004;22(12):2461-8.

60) Hurria A, Togawa K, Mohile SG, Owusu C, Klepin HD, Gross CP, et al. Predicting chemotherapy toxicity in older adults with cancer:
A prospective multicenter study. J Clin Oncol 2011;29(25):3457-65. 61) Hamaker ME, Seynaeve C, Wymenga AN, van Tinteren H, Nortier $\mathrm{JW}$, Maartense E, et al. Baseline comprehensive geriatric assessment is associated with toxicity and survival in elderly metastatic breast cancer patients receiving single-agent chemotherapy: Results from the OMEGA study of the Dutch breast cancer trialists' group. Breast 2014;23(1):81-7.

62) Denkinger MD, Hasch M, Gerstmayer A, Kreienberg R, Nikolaus T, Hancke K. Predicting fatigue in older breast cancer patients receiving radiotherapy. A head-to-head comparison of established assessments. Z Gerontol Geriatr 2015;48(2):128-34.

63) Kalsi T, Babic-Illman G, Ross PJ, Maisey NR, Hughes S, Fields P, et al. The impact of comprehensive geriatric assessment interventions on tolerance to chemotherapy in older people. Br J Cancer 2015; 112(9):1435-44.

64) Kenis C, Bron D, Libert $Y$, Decoster L, Van Puyvelde K, Scalliet P, et al. Relevance of a systematic geriatric screening and assessment in older patients with cancer: Results of a prospective multicentric study. Ann Oncol 2013;24(5):1306-12.

65) Girre V, Falcou MC, Gisselbrecht M, Gridel G, Mosseri V, Bouleuc $\mathrm{C}$, et al. Does a geriatric oncology consultation modify the cancer treatment plan for elderly patients? J Gerontol A Biol Sci Med Sci 2008;63(7):724-30.

66) Caillet P, Canoui-Poitrine F, Vouriot J, Berle M, Reinald N, Krypciak $\mathrm{S}$, et al. Comprehensive geriatric assessment in the decision-making process in elderly patients with cancer: ELCAPA study. J Clin Oncol 2011;29(27):3636-42.

67) Pottel L, Lycke M, Boterberg T, Pottel H, Goethals L, Duprez F, et al. G-8 indicates overall and quality-adjusted survival in older head and neck cancer patients treated with curative radiochemotherapy. BMC Cancer 2015;15:875.

68) Kwon M, Kim SA, Roh JL, Lee SW, Kim SB, Choi SH, et al. An introduction to a head and neck cancer-specific frailty index and its clinical implications in elderly patients: A Prospective observational study focusing on respiratory and swallowing functions. Oncologist 2016;21(9):1091-8.

69) Bras L, Peters TT, Wedman J, Plaat BE, Witjes MJ, van Leeuwen $\mathrm{BL}$, et al. Predictive value of the groningen frailty indicator for treatment outcomes in elderly patients after head and neck, or skin cancer surgery in a retrospective cohort. Clin Otolaryngol 2015;40(5):474-82.

70) Kubrak C, Olson K, Jha N, Jensen L, McCargar L, Seikaly H, et al. Nutrition impact symptoms: Key determinants of reduced dietary intake, weight loss, and reduced functional capacity of patients with head and neck cancer before treatment. Head Neck 2010;32(3):290300.

71) Paccagnella A, Morello M, Da Mosto MC, Baruffi C, Marcon ML, Gava A, et al. Early nutritional intervention improves treatment tolerance and outcomes in head and neck cancer patients undergoing concurrent chemoradiotherapy. Support Care Cancer 2010;18(7): $837-45$.

72) Nieman CL, Pitman KT, Tufaro AP, Eisele DW, Frick KD, Gourin CG. The effect of frailty on short-term outcomes after head and neck cancer surgery. Laryngoscope 2018;128(1):102-10.

73) Abt NB, Richmon JD, Koch WM, Eisele DW, Agrawal N. Assessment of the predictive value of the modified frailty index for ClavienDindo grade IV critical care complications in major head and neck cancer operations. JAMA Otolaryngol Head Neck Surg 2016;142(7): 658-64.

74) Pottel L, Lycke M, Boterberg T, Pottel H, Goethals L, Duprez F, et al. Serial comprehensive geriatric assessment in elderly head and neck cancer patients undergoing curative radiotherapy identifies evolution of multidimensional health problems and is indicative of quality of life. Eur J Cancer Care (Engl) 2014;23(3):401-12. 
Korean J Otorhinolaryngol-Head Neck Surg I 2019;62(3):141-50

\section{정답 및 해설}

답 (3)

해설 폐기능이 좋지 않아 기침이 어렵거나 뇌병변으로 인해 수술 후 재활이 어려울 경우는 환자와 보호자, 그리고 의료진과의 충분한 의사 소통을 통해 술식을 결정하는 것이 좋다. 